\title{
Dopant Quantification by Atomic-scale Energy Dispersive X-ray Analysis
}

\author{
Zhen Chen $^{1}$, Scott D. Findlay ${ }^{1}$, Matthew Weyland ${ }^{2,3}$, Adrian J. D’Alfonso ${ }^{4}$ and Leslie J. Allen ${ }^{4}$ \\ 1. School of Physics and Astronomy, Monash University, Victoria 3800, Australia. \\ 2. Monash Centre for Electron Microscopy, Monash University, Victoria 3800, Australia. \\ 3. Department of Materials Engineering, Monash University, Victoria 3800, Australia. \\ 4. School of Physics, University of Melbourne, Victoria 3010, Australia.
}

Atomic resolution energy dispersive X-ray (EDX) mapping in scanning transmission electron microscopy (STEM) has been realized recently [1, 2]. Strong dynamical scattering of electrons along the well-aligned atomic columns leads to a nonlinear relationship between X-ray counts and elemental concentration, necessitating detailed image simulation when quantification is sought [3, 4]. The introduction of dopants, a circumstance of great interest for practical applications in material science, offers further complications. For quantitative work in high angle annular dark field (HAADF) imaging, the simple fractional occupancy procedure, i.e. 'virtual crystal approximation' as depicted in figure 1(a), is not always sufficient [5]. In particular, for atomic resolution analysis, it is possible that columns with the same concentration but different depth distributions of the substitutional dopants will produce quantitatively different signals, as depicted in figure 1(b). We will explore the limits this imposes on precise concentration determination via atomic-resolution STEM EDX.

Our case study is a $\mathrm{GaAs} / \mathrm{Al}_{0.5} \mathrm{Ga}_{0.5} \mathrm{As}$ heterostructured nanowire, with preliminary experimental data on morphology shown in figure 1(c) and (d). To explore the uncertainties in the quantification of the Al due to different $\mathrm{Al}$ depth distributions, we constructed random $\mathrm{Al} / \mathrm{Ga}$ configurations after the fashion of the schematic in figure 1(b) and simulated the resultant EDX signals. Figure 2(a) shows a histogram of the simulated EDX signals for 40 such configurations for concentrations of $45 \%, 50 \%$ and $55 \%$ aluminum by atomic number in a column 40 atoms long, using $302 \mathrm{keV}$ electrons and a probe-forming aperture angle of 15 mrad.

The average Al-K signal over a unit cell from different $\mathrm{Al} / \mathrm{Ga}$ configurations shows a Gaussian-like distribution, and the mean and standard deviation are given in table 1 . It is seen that the $3 \sigma$ statistical confidence limits, i.e. uncertainty bounds within a 95\% confidence interval, overlap for the Al-K X-rays intensity from samples with $5 \%$ concentration change. In other words, it is difficult to distinguish variations in intensity based on different configurations for the same concentration from genuine differences in concentration at this level. Note too that the fractional occupancy simulation is more than $3 \%$ larger for Al-K signal.

The channeling effect can in principle be reduced by using a large probe forming aperture, though whether this is possible in practice will depend on the capability of the aberration-corrector available. Figure 2(b) and the right hand side of table 1 show the results for a 50 mrad probe-forming aperture semiangle. The Al-K X-rays intensity from 45\%, 50\%, and 55\% Al concentration is then clearly separated beyond $3 \sigma$ and, furthermore, the intensity from fractional occupancy is identical to the average intensity of different $\mathrm{Al} / \mathrm{Ga}$ depth distributions. The fractional occupancy simulation procedure is much more reliable for the larger convergence angle, a boon for composition characterization by EDX as it is much more efficient in terms of computation time [6]. 
References:

[1] AJ D’Alfonso et al, Physical Review B 81 (2010), 100101.

[2] PG Kotula et al, Microscopy and Microanalysis 18 (2012), p. 691-698.

[3] G Kothleitner et al, Physical Review Letters 112 (2014), 085501.

[4] Z Chen et al, Submitted for publication (2014).

[5] E Carlino and V Grillo, Physical Review B 71 (2005), 235303.

[6] The authors wish to thank Jenny Wong-Leung and Jenny Nian-Jiang from the Australian National University for the nanowire specimens and acknowledge support from the Australian Research Council's Discovery Projects funding scheme (Projects DP110102228 and DP140102538) and DECRA funding scheme (Project DE130100739).

(a)

(d)

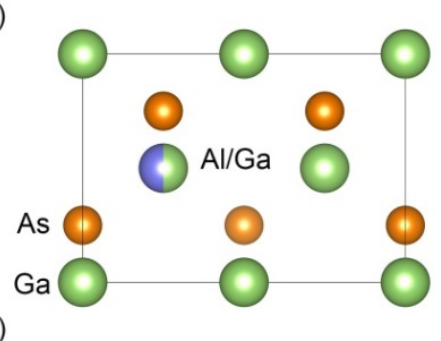

(b)

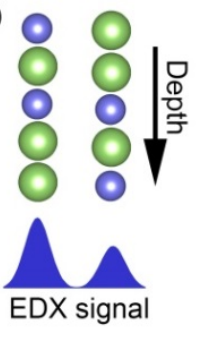

(c)

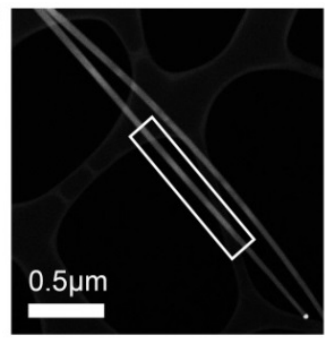

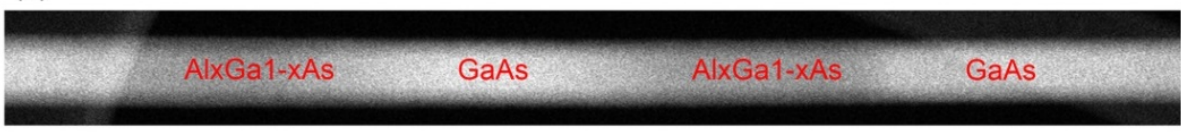

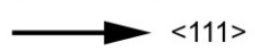

$50 \mathrm{~nm}$

Figure 1. Structure model and morphology of $\mathrm{GaAs} / \mathrm{Al}_{0.5} \mathrm{Ga}_{0.5} \mathrm{As}$ nanowire. (a). Structure model used for simulation, [110] zone-axis. (b) Schematic of possible Al/Ga depth distributions. (c) STEM-HAADF image. (d) Enlarged image of the white rectangular region in (c) showing the heterostructure.

(a)

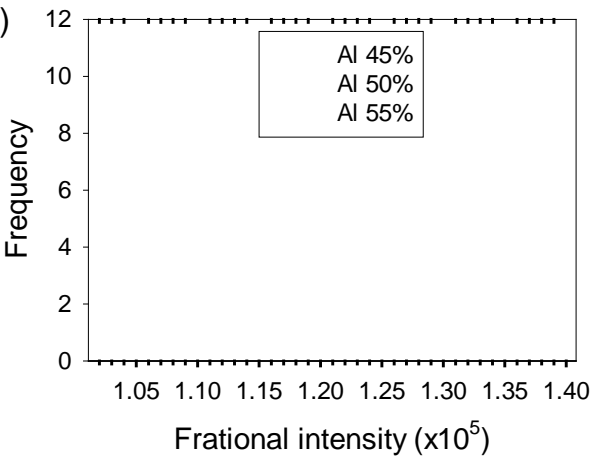

(b)

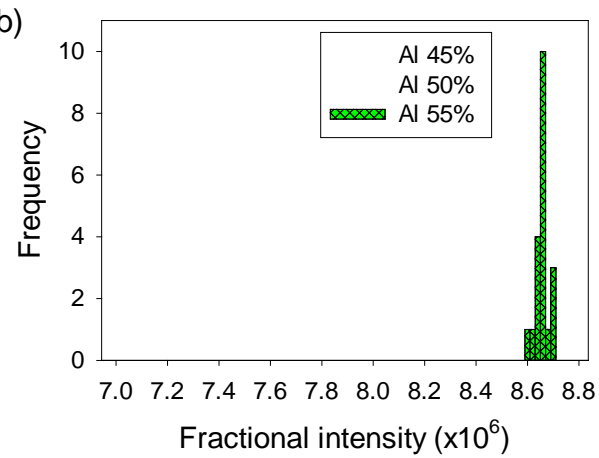

Figure 2. Histogram of Al-K X-rays intensity for different $\mathrm{Al}$ depth distributions. For a convergence angle of (a) 15 mrad and (b) 50 mrad. The legends show the Al concentration.

\begin{tabular}{|c|c|c|c|c|c|c|}
\hline & \multicolumn{5}{|c|}{ Convergence angle } \\
\cline { 2 - 7 } & \multicolumn{3}{|c|}{$15 \mathrm{mrad}$} & \multicolumn{3}{c|}{$50 \mathrm{mrad}$} \\
\hline Al content & $45 \%$ & $50 \%$ & $55 \%$ & $45 \%$ & $50 \%$ & $55 \%$ \\
\hline Mean & $1.07 \times 10^{-5}$ & $1.19 \times 10^{-5}$ & $1.32 \times 10^{-5}$ & $0.71 \times 10^{-5}$ & $0.79 \times 10^{-5}$ & $0.87 \times 10^{-5}$ \\
\hline Standard deviation & $3.79 \times 10^{-7}$ & $4.44 \times 10^{-7}$ & $3.37 \times 10^{-7}$ & $3.87 \times 10^{-8}$ & $2.68 \times 10^{-8}$ & $2.43 \times 10^{-8}$ \\
\hline Fractional occupancy & $1.11 \times 10^{-5}$ & $1.24 \times 10^{-5}$ & $1.36 \times 10^{-5}$ & $0.71 \times 10^{-5}$ & $0.79 \times 10^{-5}$ & $0.87 \times 10^{-5}$ \\
\hline
\end{tabular}

Table 1. Average intensity of Al-K X-rays across different Al/Ga depth distributions. 